\title{
Atomic Resolution Tomography of Magnetically Anisotropic FePt Nanoparticles
}

\author{
Mary Scott $^{1}$, C.-C. Chen ${ }^{1}$, Hao Zeng ${ }^{2}$, Peter Ercius ${ }^{3}$ and John Miao ${ }^{1}$ \\ ${ }^{1}$ Department of Physics \& Astronomy and California NanoSystems Institute, University of California, \\ Los Angeles, CA 90095, USA. \\ ${ }^{2}$ Department of Physics, University at Buffalo, the State University of New York, Buffalo, New York \\ 14260, USA. \\ ${ }^{3}$ National Center for Electron Microscopy, Lawrence Berkeley National Laboratory, Berkeley, \\ California 94720, USA.
}

Bimetallic FePt nanoparticles have found application in high-density magnetic memory devices due to their large uniaxial magnetic anisotropy and overall stability [1]. These particles form an ordered $\mathrm{L} 1_{0}$ state, in which the Fe and Pt segregate into alternately stacked (100) planes. While it is well known that the magnetic anisotropy in this material arises from the $\mathrm{L}_{0}$ phase [1], to truly understand the functionality of this material it is necessary to determine the atomic arrangement and defect structures in the interior of the particles. To do this, we have undertaken an atomic resolution tomographic study of 8-10nm bimetallic FePt nanoparticles in the $\mathrm{L1}_{0}$ state.

Electron tomography, which reconstructs an object's interior 3D structure based on a series of projectional images taken over a range of angles, is a natural choice to determine the 3D atomic structure of nanomaterials. However, until recently, the $3 \mathrm{D}$ resolution of electron tomography techniques has been limited to $\sim 1 \mathrm{~nm}$ [2]. Over the past several years we have developed a 3D characterization technique, termed equally sloped tomography (EST), to determine the local atomic structure of nanomaterials using high resolution electron tomography. In addition to a precision reconstruction algorithm, determining a nanomaterial's 3D atomic structure requires an accurate alignment technique and high quality, high resolution input data. Therefore we have also developed a center of mass alignment method that has been used to align the projections with atomic level accuracy [3] and acquired high resolution tilt series on both corrected and uncorrected scanning transmission electron microscopes. To reconstruct, EST then uses a Fourier-based iterative algorithm to arrive at a solution for the interior structure of the object, using constraints such as object size while enforcing the original data, but not making assumptions about the crystal structure of the object. Previously, we have acquired tilt series on an uncorrected FEI Titan to resolve both lattice structure and atomic resolution defects [3],[4].

To study the FePt particles, we have taken several atomic resolution tilt series on the National Center for Electron Microscopy's TEAM I microscope, a double-corrected FEI Titan. Samples were prepared of 8$10 \mathrm{~nm}$ FePt particles from solution, which were then dropcast and subsequently annealed to produce an $\mathrm{L} 1_{0}$ phase. Several of these particles have been imaged and reconstructed as described above. In this way, we are able to resolve local features at atomic resolution in several FePt particles (Figure 1). After reconstruction, $\mathrm{Fe}$ and $\mathrm{Pt}$ atoms can be differentiated by isolating the forbidden Bragg peaks in the 3D Fourier transform of the reconstruction. These peaks, which would not be present in a single-species face centered cubic lattice, but do appear in the $\mathrm{L}_{0}$ structure, can be used to separate the individual atomic species in the reconstruction. By differentiating between Fe and $\mathrm{Pt}$ in our reconstruction, our technique not only identifies physical defects in the crystal lattice, but also features such as antiphase boundaries that can only be identified by labeling the atomic species. Using EST to determine the 3D 
atomic structure of these particles, we can study the $\mathrm{L} 1_{0}$ state in unprecedented detail, and have identified not only structural defects, but also ordering faults in the $\mathrm{L} 1_{0}$ phase which ultimately will affect the magnetic functionality of this nanomaterial.

[1] S Sun et al., Science 287 (2000) p. 1989-1992.

[2] Midgley, P. A. \& Weyland, M. STEM Tomography. In Scanning Transmission Electron

Microscopy: Imaging and Analysis. ed. Pennycook, S. J. \& Nellist, P. D. p. 353-392 (Springer, 2011).

[3] M. C. Scott, et al., Nature 483 (2012) p. 444-447.

[4] C.-C. Chen, et al., Nature 496 (2013) p. 74-77.

[5] This work is partially supported by U. S. Department of Energy, Grant \#: DE-FG02-13ER46943.

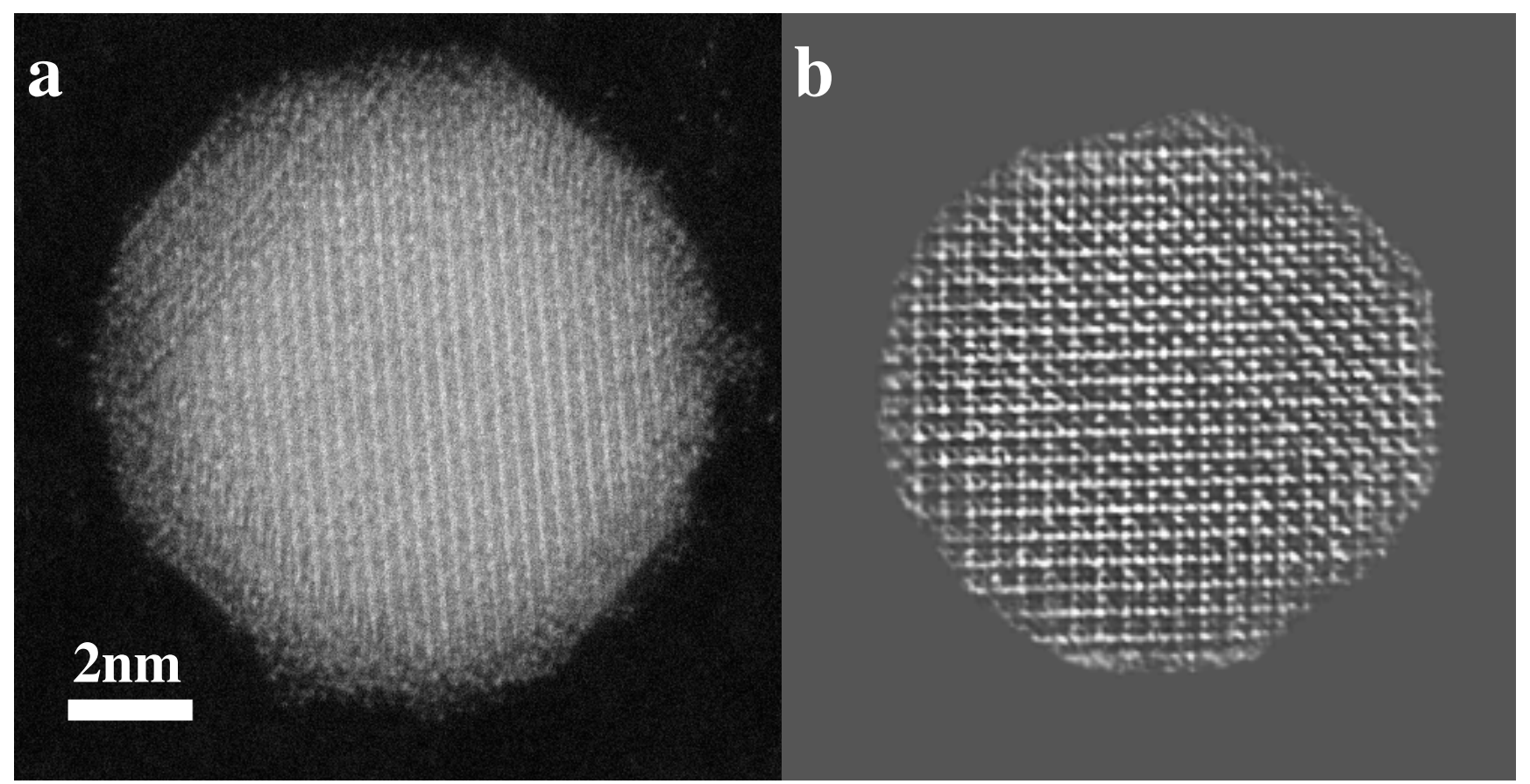

Figure 1. A representative FePt particle and a slice through its reconstruction. a, An experimental projection of an FePt nanoparticle, taken in the TEAM I microscope at the National Center for Electron Microscopy as part of a tomographic tilt series. b, A $0.3 \mathrm{~nm}$ thick slice through a portion of the reconstruction of the particle in (a). Individual atoms are clearly visible. 